\title{
Finite Element Modelling of Mechanical Phenomena Connected to the Technological Process of Continuous Casting of Steel
}

J. Heger

\begin{abstract}
A finite element method algorithm is presented that enables numerical simulation of real phenomena that take place during an industrial process of continuous casting of steel. The algorithm takes into account all known kinds of nonlinearities: material nonlinearity connected to the nonlinear temperature dependence of material properties, large deformations from the process of material forming and contacts between the slab and rollers of the strand. Received results describe the sensitivity of the product to crack initiation, not only during the process of continuous casting itself but also in the finished and cooled slab.
\end{abstract}

Keywords: Continuous casting, steel slab, numerical simulation, finite element method, nonlinearity, large deformations, contact, crack initiation.

\section{Notation}

c $\quad\left[\mathrm{J} \mathrm{kg}^{-1} \mathrm{~K}^{-1}\right]$

specific heat

$t \quad[\mathrm{~K}]$

temperature

$x, y, z \quad[\mathrm{~m}]$

coordinates

$Q \quad\left[\mathrm{~W} \mathrm{~m}^{-3}\right]$

heat source

$\lambda \quad\left[\mathrm{W} \mathrm{m}^{-1} \mathrm{~K}^{-1}\right]$ thermal conductivity

$\rho \quad\left[\mathrm{kg} \mathrm{m}^{-3}\right] \quad$ density

$\tau \quad[\mathrm{s}] \quad$ time

\section{Introduction}

The transition from the conventional pouring of steel into metal moulds to the progressive technology of continuous casting is progressing throughout the world. In the first stage of continuous casting the liquid metal flows from a ladle through the reservoir (tundish) into a water-cooled copper mould. Its goal is to remove such an amount of the heat from the melt that a thick enough layer of solidified metal is created on its surface (primary cooling zone) to contain the liquid metal. The slab sinks down gradually and after exiting the mould is taken off by the roller conveyor and sprayed by the system of water and air-water nozzles (secondary cooling zone). The natural convection and radiation of the surrounding air (tertiary cooling zone) further cool it. The endless product is divided into pieces of desired length using the torch cut-off technique after the complete solidification of its core. Received slabs are freely cooled under the natural air conditions. The scheme of a radial slab caster with its main parts and used terminology can be seen on Fig. 1 .

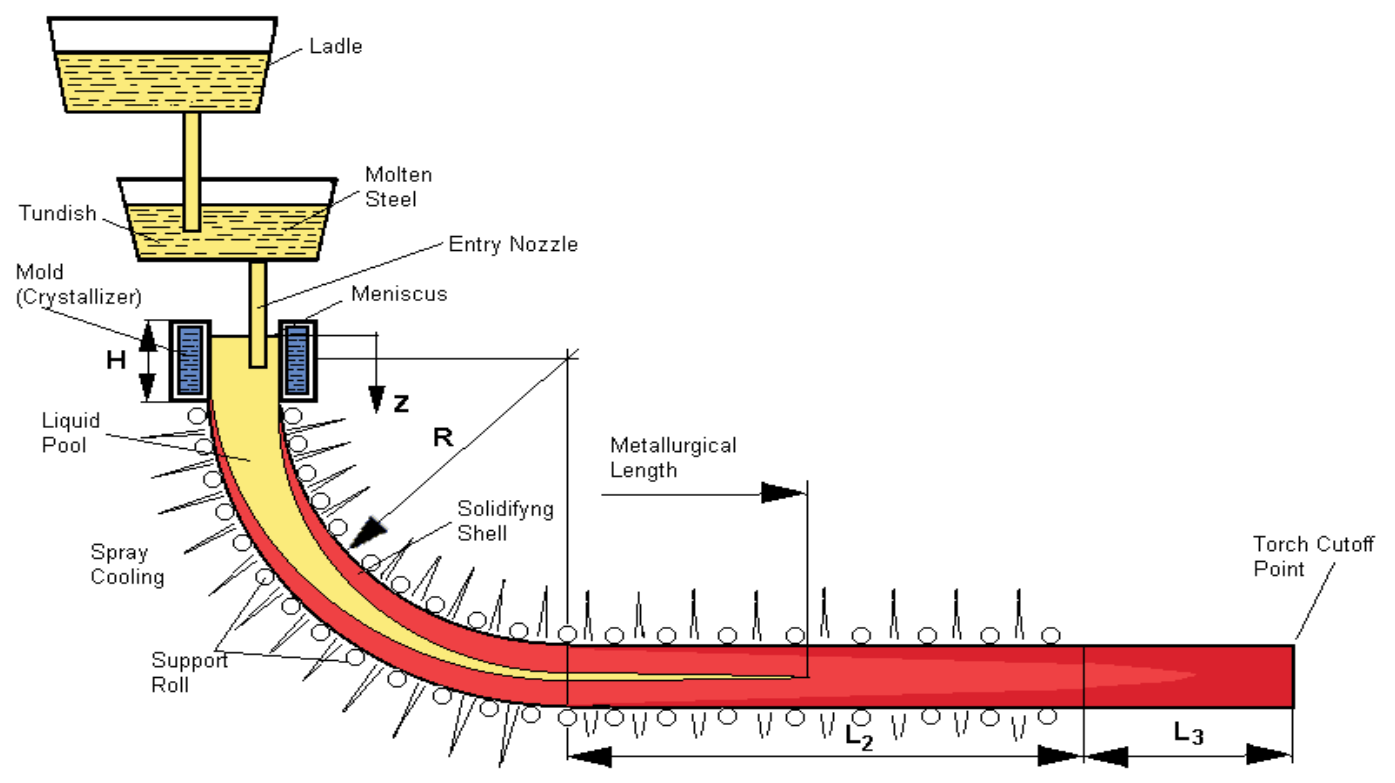

Fig. 1: Main parts of a radial slab caster 
One of the problems in the progressive technology of continuous casting is the formation of cracks in slabs. The main cause of cracks is the development of stresses, mechanical and thermal, during slab solidification and cooling. Cracks form when this stress exceeds the local strength of material, i.e., when the corresponding strain exceeds the local material ductility.

The operating parameters like pouring temperature, casting rate, the length and taper of the mould, cooling intensity in the mould and in the zones of secondary and tertiary cooling, influence the tendency of the slab to create cracks. They effect the tendency to crack forming in two ways:

- the direct impact influencing the distribution of stresses and strains during the continuous casting process,

- the indirect impact influencing the change of metallographical structure.

The aim of the work was to design and tune an algorithm that would simulate numerically the substantial phenomena that take place during the process of continuous casting. The main consideration was the direct impact of outer impulses on the evolution of stress-strain distribution and on the sensitivity of the slab to the formation of surface and subsurface defects.

\section{Physically mathematical analysis of the process of the continuous casting of steel}

\subsection{Coupled thermo-structural transient problem}

Continuous casting of steel is from the physical point of view a coupled thermo-structural problem. It is described by the partial differential equations containing thermal and mechanical variables. Theoretically these two groups of variables are interdependent; the temperature distribution influences the mechanical response of the continuum and vice versa. However with respect to the relatively small rate of deformation at the process of continuous casting, it is possible to assume on the technical level of distinguishability that the mechanical phenomena will not significantly influence the temperature distribution during the process of continuous casting. Assuming this, it is possible to decouple the thermal and mechanical processes. The thermal processes are considered to be of primary importance and to influence the mechanical processes in the solidifying and cooled half-finished product.

Thermal impact can show itself in two ways:

1) by the thermal expansion of material,

2) by the varying mechanical properties of material as a result of their temperature dependence.

\subsection{Numerical simulation of thermal phenomena in the solidifying slab}

In conventional gravitation pouring of castings the casting is cooled by the mould and ambient atmosphere. From the point of view of thermo-kinetics, it is a case of three dimensional transient heat and mass transfer in the system casting-mould-surroundings. The problem of casting solidifi- cation and cooling in conventional gravitation pouring can be described by the Fourier differential equation

$\frac{\partial}{\partial x}\left(\lambda_{x} \frac{\partial t}{\partial x}\right)+\frac{\partial}{\partial y}\left(\lambda_{y} \frac{\partial t}{\partial y}\right)+\frac{\partial}{\partial z}\left(\lambda_{z} \frac{\partial t}{\partial z}\right)+Q=\rho c \frac{\partial t}{\partial \tau}$.

Corresponding initial conditions for the whole region and boundary conditions for all surfaces during the whole process must be known for the problem to be defined in the correct way.

Mathematical description of the solidification and cooling of the slab during continuous casting is a slightly more complicated problem. It is a transport problem with the transfer of heat and mass that can be described by the Fourier-Kirchhof equation

$$
\begin{aligned}
\rho c\left(\frac{\partial t}{\partial \tau}+w_{z} \frac{\partial t}{\partial z}\right) & =\frac{\partial}{\partial x}\left(\lambda_{x} \frac{\partial t}{\partial x}\right)+\frac{\partial}{\partial y}\left(\lambda_{y} \frac{\partial t}{\partial y}\right)+ \\
& +\frac{\partial}{\partial z}\left(\lambda_{z} \frac{\partial t}{\partial z}\right)+Q
\end{aligned}
$$

assuming, for simplicity, that the motion of the slab is in the direction of the $z$ coordinate axis at velocity $w_{z}$.

\subsection{Motion of the continuously cast slab}

Relative motion of the slab with respect to the mould and the caster during the process of continuous casting may be included in the numerical simulation of thermal phenomena in two ways:

1) by fixing the boundary conditions and moving the slab the transport problem solution described by the Fourier-Kirchhof equation,

2) by fixing the slab and moving the boundary conditions the problem solution is described by the Fourier equation.

Both these approaches produce identical results. Solution using the Fourier-Kirchhof equation requires an unusual algorithm but is a fluent and less demanding way of manipulating the input data using boundary conditions that do not change with time or space. On the other hand solution using the Fourier equation does not require a special algorithm but entering boundary conditions moving in time means a huge amount of data, which can cause considerable technical difficulties in running the program. The first approach has been chosen for its simplicity with the input data manipulation.

\subsection{Finite difference method application for the numerical simulation of thermal phenomena during continuous casting of steel}

For the thermal phenomena simulation of the continuous casting of steel a specialised program was created on the principal of the finite difference method [1]. This program solves the Fourier-Kirchhof equation for transient thermal problems of continuously cast steel slabs with the width 800 to $1600 \mathrm{~mm}$ and the thickness 120 to $250 \mathrm{~mm}$. The program works with the explicit version of the finite difference method using the smooth thermal dependence of enthalpy. It is equipped with the original mesh generator as well with as a graphical post-processor. There is a user friendly way of changing all the input data: caster parameters, thermo-physical parameters of 
cast material, heat transfer coefficients, slab dimensions as a whole and also dimensions of meshed elementary volumes.

With respect to the need for a subsequent stress analysis, to be performed by the finite element method following the thermal analysis, the thermal program is equipped with a finite element mesh generator that corresponds to the finite difference method mesh. It produces also the transient thermal result output, which is one of the inputs for the following structural analysis. All the transferred data are created in the format used by the finite element method program ANSYS.

\subsection{Numerical simulation of mechanical phenomena during continuous casting of steel}

Mechanical phenomena acting on the solidifying and cooled slab during the process of continuous casting of steel are simulated [2] using the finite element program system ANSYS. In this thermally affected elasto-plastic stress-strain analysis all the material properties are strongly temperature dependent. This analysis takes into account all the impacts that the slab experiences during the process of continuous casting. The originally vertical position of the slab in the mould at the beginning of the process is changed during its progress through the forming rollers of the caster to the horizontal position. The product is exposed not only to the thermal influence varying in time but also to the transport and forming at the contact with the individual rollers. In the radial part of the caster there is forming by the bending influence of the rollers, and in the following straight part there is straightening of the product which was bent in the previous part.

The described and designed simulation of mechanical phenomena during continuous casting can be used to predict the sensitivity of the product to the forming of surface and inner defects. Using the parameter called the normalised stress, defined as the ratio of the local maximum principal stress and the local yield stress, it is possible to quantify the sensitivity of the product to defects forming during the whole process of continuous casting. It describes this sensitivity from the pouring of the melt, through the solidification and cooling of the product till the equilibration of the product temperature with the ambient temperature. Received results corresponding to this last described status predicate the distribution of residual stresses in the product after the process of continuous casting is finished.

The advantage of numerical simulation of continuous casting is that, after the algorithm is designed and tuned, it predicts the sensitivity of the product to the forming of inner and surface defects at various thermal and mechanical conditions of the cast material. These conditions comprise, for example, varying cast profiles, pouring temperature, casting rate, shape of caster, varying rollers distribution and their adjustment, influence of changing the cooling intensity.

\subsection{Finite element algorithm for numerical simulation of mechanical phenomena proceeding during the process of continuous casting of steel}

Taking into account the high requirements for computer memory, available disc space and time of the computation, the problem of numerical simulation of mechanical phenomena during the process of continuous casting of steel was in the first stage drawn as a two dimensional problem. For the slab with a rectangular cross section $1530 \mathrm{~mm} \times 250 \mathrm{~mm}$, a longitudinal plane section was chosen to represent the solution in the plane of symmetry with the plane strain assumption. The cross section was meshed using quadrilateral finite elements. The program system ANSYS version 5.7 was used for the finite element solution.

Besides the slab itself the finite element model also comprises 128 rollers of six diameters (Fig. 2). The distribution and adjustment of these rollers creates the shape of the caster

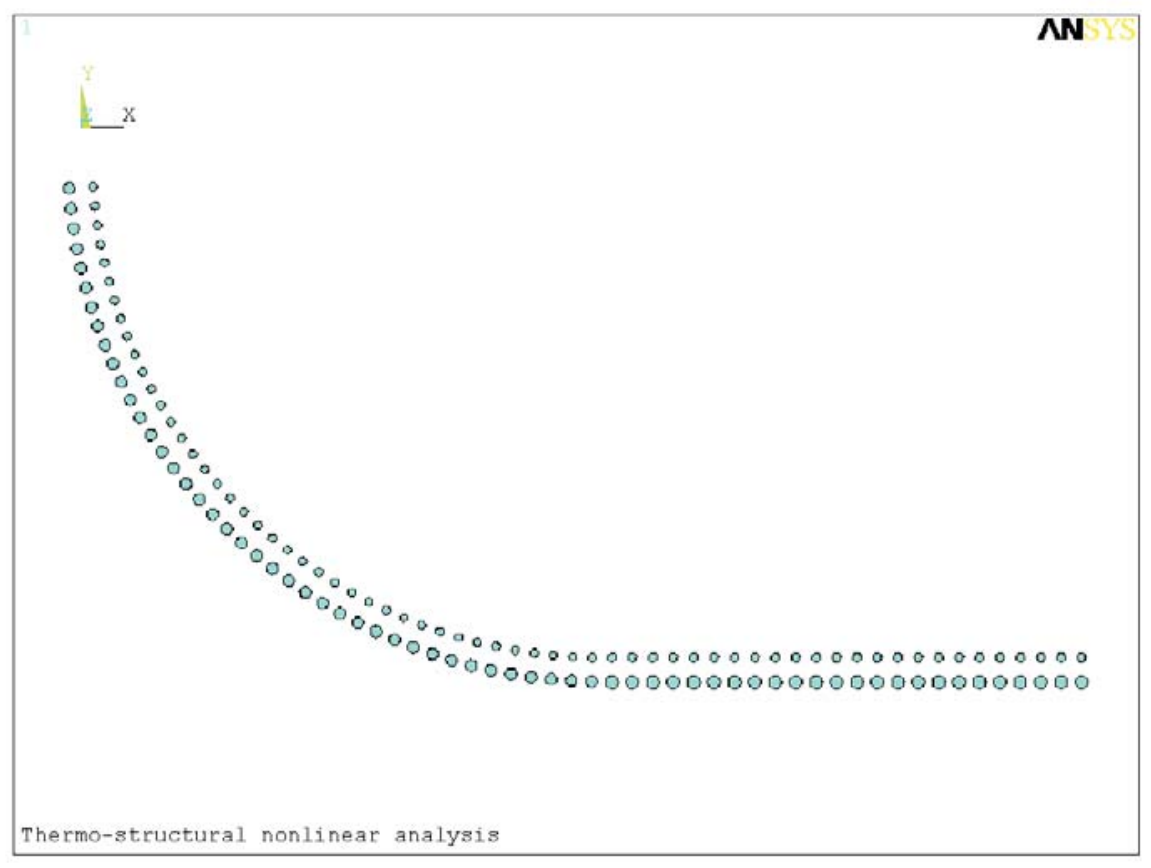

Fig. 2: Roller distribution at the strand of continuous casting 


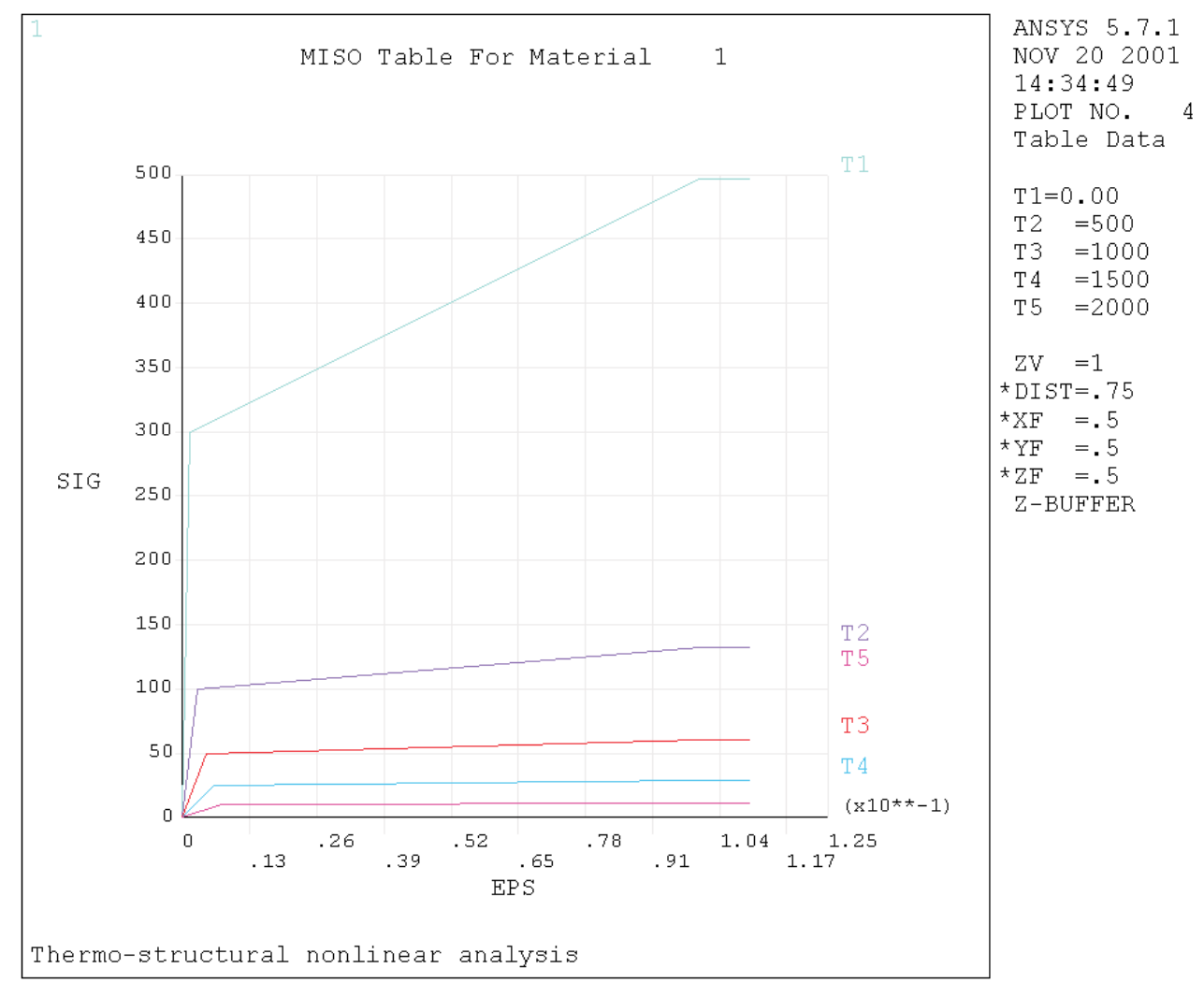

Fig. 3: Stress-strain curves

and by this way they also affect the final shape of the product. In the finite element contact analysis these rollers are meshed as the target elements TARGET169. For the slab meshing visco-plastic elements VISCO106 have been used. The material properties of the manufactured slab are strongly temperature dependent. Their thermal stress-strain dependence curves can be seen on Fig. 3 .

The applied regular quadrilateral mesh is designed with 23 elements along the slab height to enable a detailed description of the stress and strain gradients. The basic element dimensions are $10.870 \mathrm{~mm} \times 50.192 \mathrm{~mm}$. The direction of the longer dimension corresponds to the direction of the slab longitudinal axis. The resulting finite element mesh contains 21827 elements with $231 \times 84$ nodes. The nodes on the external surface of the product are connected to the target elements by means of 1898 contact elements CONTA172. The connection of target and contact elements enables the simulation of real contact conditions during the process of continuous casting.

The simulation algorithm of the real process of continuous casting is based on the following principle.

The melted steel reservoir is modelled by a vertical strip of elements of material at the melting temperature. The remaining part of the manufactured product is of material with the temperature distribution found in the previous numerical analysis based on the finite difference method. However, this part of the product is used in the model only as a dummy part helping to accommodate contact nonlinearities in the fully developed contact conditions. Results received from this re- gion cannot be used for the evaluation of the real stress-strain circumstances in the manufactured product. Each element and each node of the vertical region that is filled by the melted metal at the beginning of the process has to experience its own unique thermal-stress-strain transient history during the passage of the whole product through the caster created by the adjusted pairs of rollers. The algorithm enables simulation of the real passage of the manufactured product through the rolling mill of the caster, during which the plastic strain in every individual location of the product is accumulated.

For the numerical simulation of mechanical phenomena proceeding during the process of continuous casting it is not enough to mesh the problem in space but it must be meshed also in time. For the numerical solution, a time step was applied that corresponded to the transport of the product by the length of one element $(50.192 \mathrm{~mm})$. The algorithm is designed so that during the simulation the whole originally melted product in a total length of $24.594 \mathrm{~m}$ passes through the pairs of rollers. A fictitious time difference of 1 second is added to the global time at each finished step of the analysis. Altogether 482 time steps were employed for the continuous casting simulation.

Potentially critical locations can be revealed by graphical evaluation of results for the whole product. An example of the many evaluated results - distribution of stress components along the outer surface of the product - is shown in Fig. 4. Waves corresponding to the straightening of the previously bent product can be seen on the second half of curves representing axial (SY) and transversal (SZ) stress components. 


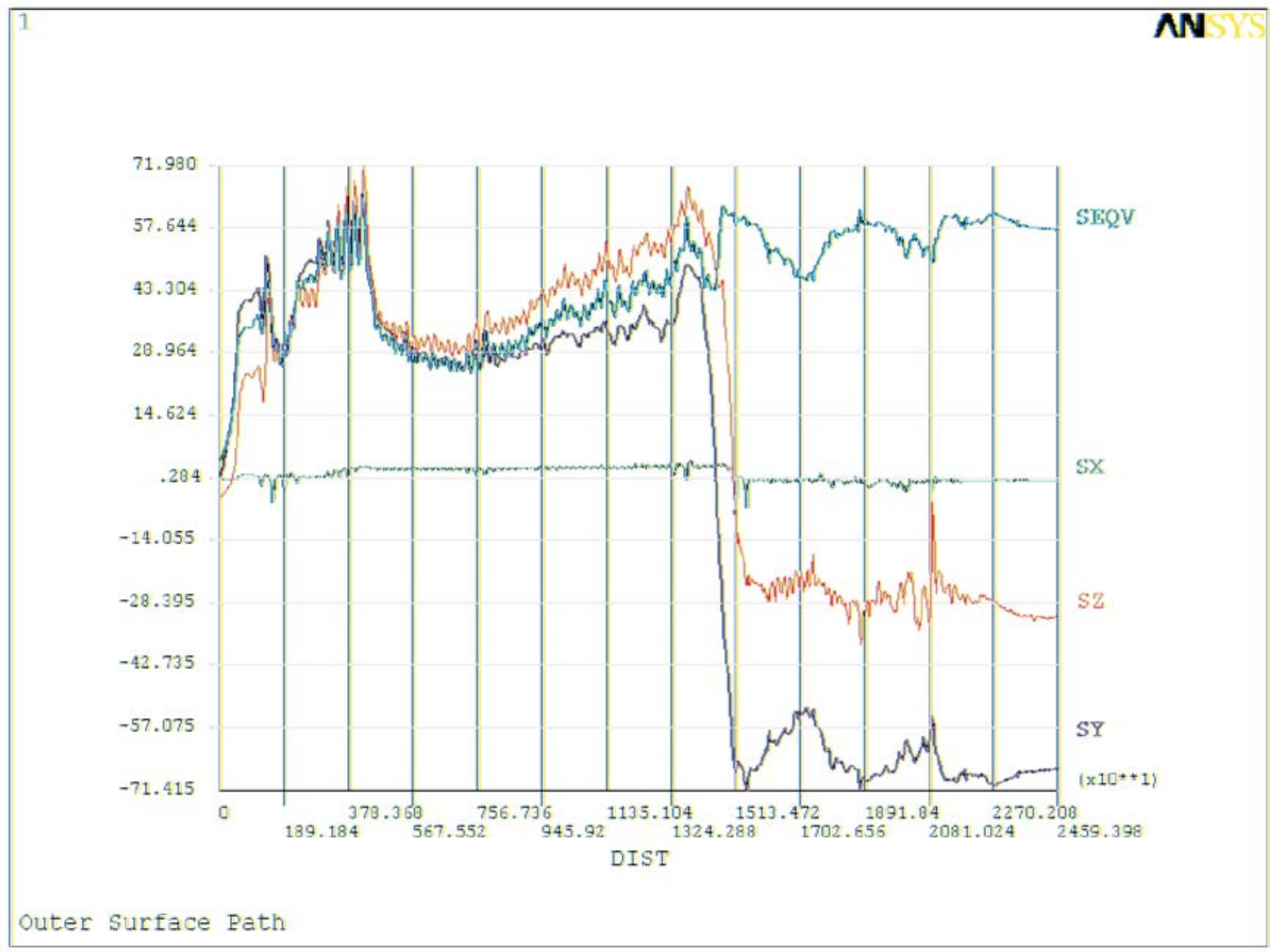

Fig. 4: Stress distribution along the product outer surface

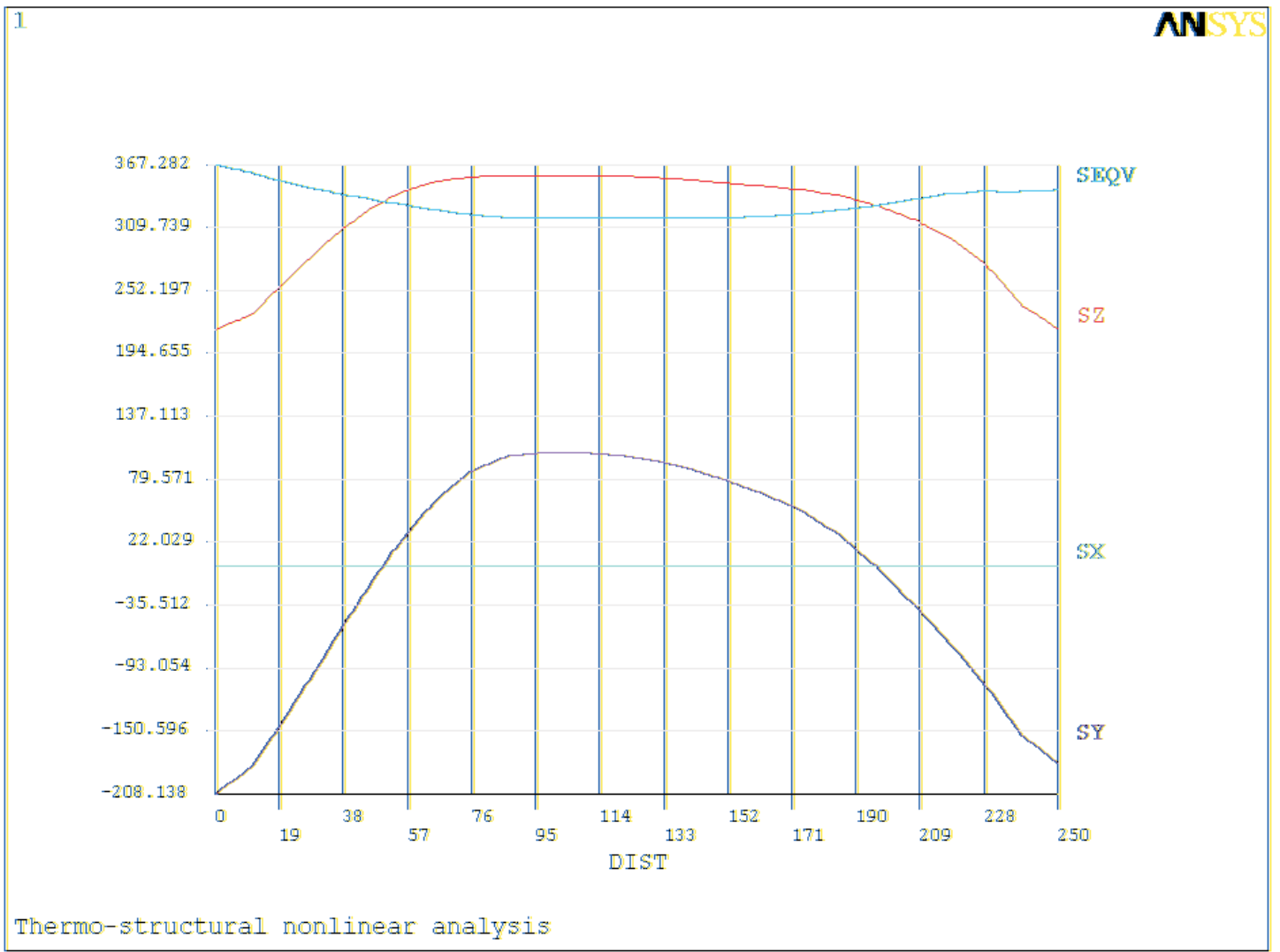

Fig. 5: Residual stress distribution

(C) Czech Technical University Publishing House http://ctn.cvut.cz/ap/ 


\subsection{Residual stresses}

The endless product of continuous casting is torch cut-off into slabs of the prescribed length and their successive gradual cooling in the environment of the production hall follows. It is a long-time process of natural convection taking several days. After the temperature equilibration the slab adopts the ambient temperature. Distribution of residual stresses through the thickness of the final slab, the presence of which is a result of plastic strains during the technological process of continuous casting, can be seen on Fig. 5. While there are compressive axial surface stresses (SY), the transverse stresses (SZ) have the tensile character.

\section{Conception of using results of numerical simulation}

It is obvious that the higher the values of the normalised stresses that are achieved, the higher the danger of crack initiation that can be expected. However, it is generally difficult to determine the ultimate value of normalised stresses that are dangerous for the formation of defects. It is known that tensile stresses have the main damaging effect on crack initiation. This is the basic reason why the normalised maximum principal stress is used to evaluate the sensitivity of the product to crack initiation.

Since there are known locations of defect occurrence from field experience, the limiting value of normalised stresses can be determined by the calibration of the numerical simulation results. The main idea of this calibration is that the numerical simulation of continuous casting for the given operation conditions is performed and the limit and kind of normalised stresses is determined at which defects under the same conditions occur according to the location of the defects.

Using the statistical evaluation of more simulated processes more general material data will be received. They will enable the safety of operational conditions for the changed or newly designed parameters of the continuous casting process to be evaluated. Eliminating sets of parameters that lead to crack formation, it will be possible to derive sets of parameters under which the cracks will not occur. All the variables influencing the process of continuous casting may be considered in this process.

\section{Conclusion}

Numerical simulation of the continuous casting process contributes to the understanding of the influence of operational conditions and material properties on the development of stresses and strains during the steel manufacturing process. Identifying operational parameters that will not lead to cracks forming in the product will raise the productivity of the continuous steel casting process. Material properties and operating conditions can be chosen that will lower the slab sensitivity to crack initiation and hot tearing.

\section{Acknowledgment}

This analysis was conducted using a program devised within the framework of the GA CR projects (No. $106 / 01 / 1464,106 / 01 / 1164,106 / 01 / 0379,106 / 01 / 0382)$ of the COST-APOMAT-OC526.10, EUREKA No.2716 COOP, KONTAKT (No. 2001/015 and No.23-2003-04) and CEZ $323001 / 2201$.

\section{References}

[1] Kavicka F. et al: Two Numerical Models of a Continuously Cast Steel Slab. IV ${ }^{\text {th }}$ European Conference on Continuous Casting, Birmingham (UK), October 2002.

[2] Heger J.: Finite Element Simulation of Continuous Casting with Respect to the Stress-Strain Distribution. Research Report, University of Pittsburgh, USA, November 2001.

Dr. Jaromir Heger

phone: 01162845556

Fax: 01162845468

e-mail: jaromir.heger@power.alstom.com

ALSTOM Power Technology Centre

Mechanical Integrity

Cambridge Road, Whetstone

LE8 6LH Leicester

England, U.K. 